\title{
Measuring the Complex Permittivity of Thin Grain Samples by the Free-Space Transmission Technique
}

\author{
Jochem Roelvink, Member IEEE \\ United States Department of Agriculture, Agricultural \\ Research Service \\ Richard B. Russell Agricultural Research Center \\ Athens, GA 30605 USA \\ Jochem.Roelvink@ars.usda.gov
}

\author{
Samir Trabelsi, Fellow IEEE \\ United States Department of Agriculture, Agricultural \\ Research Service \\ Richard B. Russell Agricultural Research Center \\ Athens, GA 30605 USA \\ Samir.Trabelsi@ars.usda.gov
}

\begin{abstract}
In this paper, a numerical method for solving a higherorder model that relates the measured transmission coefficient to the permittivity of a material is used to determine the permittivity of thin grain samples. A method for resolving the phase ambiguity of the transmission coefficient is presented. Results calculated by using the higher-order model for the permittivity of grain over a range of moisture contents and frequencies are presented and compared to those calculated by using an approximate model. The advantage of using relatively thin grain samples is that a wider range of grain moisture contents can be reliably measured for a fixed sample thickness.
\end{abstract}

Keywords-Dielectric properties; permittivity measurements, microwave sensing; transmission measurements; phase-shift ambiguity.

\section{INTRODUCTION}

For processing agricultural products it is generally necessary to determine certain quality attributes of the produce. For grain, the moisture content is one of the most important material parameters. Grain moisture content can vary widely, from approximately $30 \%$ (wet basis) when harvested to $8 \%$ when packaged. The moisture content of grain is strongly correlated with the relative complex permittivity, $\varepsilon=\varepsilon^{\prime}-\mathrm{j} \varepsilon^{\prime \prime}$. By measuring the dielectric properties of grain, the moisture content can be determined.

Over the years, many nondestructive techniques have been developed for measuring the dielectric properties of materials in the microwave band [1]. These can be broadly categorized as techniques based on the measurement of complex reflection coefficient [2], complex transmission coefficient [3] (or both [4]), and resonance techniques for measuring the dielectric properties of low-loss (small $\left.\varepsilon^{\prime \prime}\right)$ materials [5]. Free-space transmission based microwave sensors are capable of accurate measurements for low to medium $\varepsilon^{\prime}$ agricultural products, with low to medium loss, such as grain [3]. Additionally, they have the advantage of requiring minimal sample preparation. These sensors generally operate by measuring the attenuation and phase shift of an electromagnetic wave that propagates through the dielectric material. This vector measurement can be used to estimate the complex transmission coefficient, from which the permittivity of the material can be calculated.
In many previous studies, a minimum signal attenuation of $10 \mathrm{~dB}$ through the sample has been required $[1,3]$, since for these situations an approximate model that relates the transmission coefficient to the permittivity can be used. However, a grain sample of a fixed thickness that has $10 \mathrm{~dB}$ attenuation in its low-moisture state can have greater than 40 $\mathrm{dB}$ attenuation in its high-moisture state. For high moistures, the attenuation can often be comparable to the noise floor of the receiver and significantly degrade the measurement accuracy. This problem can be solved by setting a maximum sample thickness for a given range of moisture contents. While this overcomes the problems associated with large signal attenuation, for low moistures the attenuation will be considerably less than $10 \mathrm{~dB}$, and the approximate model relating the transmission coefficient to the permittivity no longer gives good results. For such situations, it is necessary to solve a higher-order model for the transmission coefficient that takes into account the multiple reflections within the sample.

In this paper, a method for numerically solving the higherorder model for the transmission coefficient is used to calculate the permittivity of thin grain samples. This method includes an approach for resolving the so-called "phase ambiguity" associated with transmission based measurements [6]. Results are presented for the permittivity of grain for a range of moisture contents. These results are calculated by using the approximate and higher-order models for the measured transmission coefficient. By solving the higher-order model when the attenuation is less than $10 \mathrm{~dB}$ and the approximate model when the attenuation is greater than $10 \mathrm{~dB}$, a wider range of grain moisture contents can be determined for a fixed sample thickness.

\section{THEORY}

When an electromagnetic plane wave is transmitted through a dielectric material of thickness $d$ placed in air, as shown in Fig. 1., the complex transmission coefficient $\tau$ for perpendicular incidence can be expressed as [1]

$$
\tau=\frac{\left(1-\Gamma^{2}\right) \mathrm{e}^{-\gamma \mathrm{d}}}{1-\Gamma^{2} \mathrm{e}^{-2 \gamma d}}
$$


where $\gamma=\alpha+\mathrm{j} \beta$ is the complex propagation coefficient, $\alpha$ is the attenuation constant $(\mathrm{np} / \mathrm{m})$ and $\beta$ is the phase constant $(\operatorname{rads} / \mathrm{m}) . \Gamma$ is the complex reflection coefficient at the airdielectric interface, which can be expressed as

$$
\Gamma=\frac{\mathrm{j} \beta_{0}-\gamma}{\mathrm{j} \beta_{0}+\gamma}
$$

where $\beta_{0}=2 \pi / \lambda$ is the free-space phase constant, and $\lambda$ is the free-space wavelength. It can be shown that the complex permittivity $\varepsilon$ is related to the propagation coefficient $\gamma$ by

$$
\varepsilon=-\left(\frac{\gamma}{\beta_{0}}\right)^{2}
$$

By measuring $\tau$ with a suitable experimental setup, $\gamma$ can be determined from (1), from which $\varepsilon$ can be calculated with (3). However, since $\gamma$ cannot be expressed as an analytic function of $\tau$, in many previous studies it has been common to assume that $\Gamma=0$. This is known to be a reasonable approximation for materials with small $\varepsilon^{\prime}$ and low to medium loss if the total sample attenuation is greater than $10 \mathrm{~dB}$ [1, 3]. If this approximation is used, the complex transmission coefficient reduces to

$$
\tau=\mathrm{e}^{-\gamma \mathrm{d}}
$$

and the determination of $\gamma$ is considerably simplified.

Despite this simplification, the situation is complicated somewhat by the fact that there are an infinite number of $\gamma$ values that can satisfy (4). For example, solving (4) for $\gamma$ we have

$$
\gamma=-\frac{\ln (\tau)}{d}+\mathrm{j} \frac{2 \pi n}{d}
$$

Any integer value of $n$ in (5) will satisfy (4) (but result in different $\varepsilon$ ). This phase-shift ambiguity results because it is impossible to determine how many complete rotations in phase the wave has experienced from a single measurement of the transmission coefficient. However, as proposed [6], assuming that $\varepsilon$ is a slowly varying function of wavelength, if two measurements, $\tau_{1}$ and $\tau_{2}$, are made at two close but different wavelengths, the relationship

$$
\gamma_{1}=\gamma_{2}\left(\frac{\lambda_{2}}{\lambda_{1}}\right)
$$

can be used to determine the correct value of $n$ in (5). (The subscripts in (6) refer to each measured wavelength.)

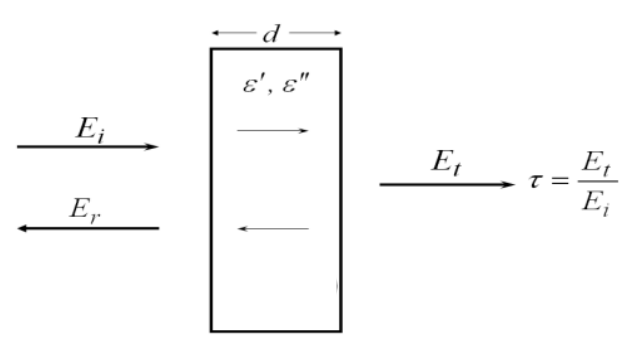

Figure. 1. Idealized representation of a free-space transmission sensor.

In order to solve the higher-order model for the propagation coefficient given by (1), we can require that $\tau_{\text {meas }}-\tau(\gamma)=0$ and solve for $\gamma$ using a suitable numerical technique (e.g. the Newton-Raphson method). The numerical solution will depend on the initial 'guess' value for $\gamma$ since, as with (4), there are an infinite number of solutions for the propagation coefficient that satisfy (1). These solutions are not related by the simple expression (5). However, (6) must still be valid if $\varepsilon$ is a slowly varying function of wavelength. So to resolve the phase-shift ambiguity when solving (1), several solutions are numerically computed for $\gamma$ at two different wavelengths and the correct solution is the one that satisfies (6).

\section{RESULTS AND DISCUSSION}

Experimental measurements were made on grain (hard red winter wheat) for a range of moisture contents between $10.3 \%$ and $22.0 \%$ (wet basis), for two sample thicknesses, $2.5 \mathrm{~cm}$ and $8.9 \mathrm{~cm}$, over the frequency range $3-17 \mathrm{GHz}$ using two horn-lens focused-beam antennas and an HP8510C ${ }^{1}$ vector network analyzer. This experimental setup has been described in detail [7]. The network analyzer was calibrated with a response-type calibration, where the reference values of the transmission coefficient were set with an empty sample holder between the antennas. Time-domain gating was applied after calibration to remove effects of post-calibration mismatches and multi-path transmission.

The measured transmission coefficient for each sample was used to determine $\varepsilon$ by the approach described in Section II. For the $8.9-\mathrm{cm}$ samples the attenuation was generally greater than $10 \mathrm{~dB}$ and (4) was solved directly, while for the $2.5-\mathrm{cm}$ samples both (1) and (4) were solved. In both cases the phase ambiguity was resolved using (6). As an example, Table I presents numerical results for $\left|\gamma_{1}\right|$ and $\left|\gamma_{2}\left[\lambda_{2} / \lambda_{1}\right]\right|$ for several solutions that satisfy (1) for a 2.5 -cm grain sample with $22.0 \%$ moisture content measured at $f_{1}=8 \mathrm{GHz}$ and $f_{2}=9 \mathrm{GHz}$. It is clear from this table that the correct solution for this case is $\left|\gamma_{1}\right|=323.7$. The slight difference in $\left|\gamma_{1}\right|$ and $\left|\gamma_{2}\left[\lambda_{2} / \lambda_{1}\right]\right|$ is due to the difference in $\varepsilon$ at the two measured frequencies.

\footnotetext{
${ }^{1}$ Mention of company or trade names is for purpose of description only and does not imply endorsement by the US Department of Agriculture.
} 
TABLE I. NUMERICAL SOLUTIONS TO (1)

\begin{tabular}{|c|c|c|}
\hline Solution & $\left|\gamma_{1}\right|$ & $\left|\gamma_{2}\left[\lambda_{2} / \lambda_{1}\right]\right|$ \\
\hline 1 & 67.0 & 96.7 \\
\hline $\mathbf{2}$ & $\mathbf{3 2 3 . 7}$ & $\mathbf{3 2 0 . 0}$ \\
\hline 3 & 573.0 & 543.3 \\
\hline
\end{tabular}

In Fig. 2, results are presented for $\varepsilon$, divided by sample density, $\rho$, as a function of moisture content for the two sample thicknesses at $6 \mathrm{GHz}$. If the approximate model (4) is used to calculate $\varepsilon$, the difference between the $2.5-\mathrm{cm}$ and $8.9-\mathrm{cm}$ samples is significant, particularly for $\varepsilon^{\prime \prime}$. For this case, the attenuation of the 2.5 -cm sample varies from $2.72 \mathrm{~dB}$ (10.3\% moisture content) to $5.92 \mathrm{~dB}$ (22.0\% moisture content), and it is therefore necessary to include the effects of the reflections at the air-dielectric interfaces. If the permittivity of the $2.5-\mathrm{cm}$ sample is calculated with (1), there is generally good agreement between the $8.9-\mathrm{cm}$ and $2.5-\mathrm{cm}$ samples.

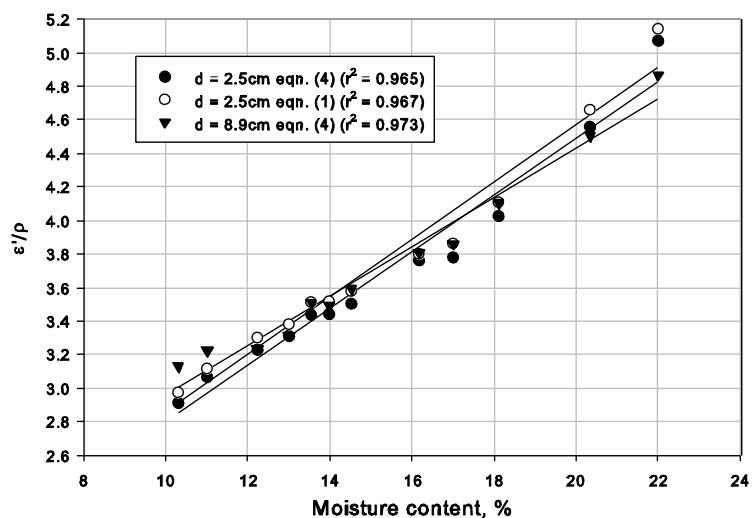

(a)

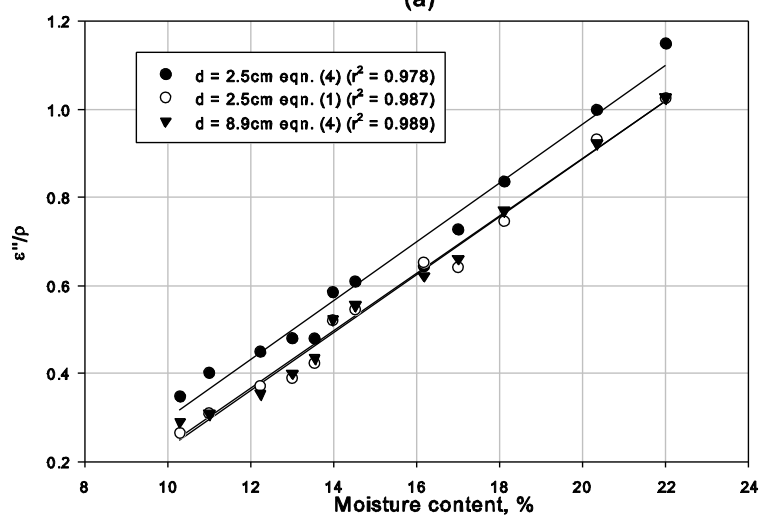

(b)

Figure 2: Variations of $\varepsilon^{\prime} / \rho$ (a) and $\varepsilon^{\prime \prime} / \rho$ (b) with moisture content for 2.5-cm and 8.9-cm grain samples at $6 \mathrm{GHz}$, determined with either (1) or (4).

In Fig. 3, results are presented for $\varepsilon$ (divided by $\rho$ ) as a function of moisture content for the two sample thicknesses at $10 \mathrm{GHz}$. In this case the attenuation of the $2.5-\mathrm{cm}$ sample varies between $5.10 \mathrm{~dB}$ and $13.55 \mathrm{~dB}$, and the differences in the permittivity calculated with either (1) or (4) are small. Comparing the results for the two sample thicknesses, it can be seen that for low grain moisture the difference in the calculated $\varepsilon$ is small. However, at $22.0 \%$ moisture content there is significant difference, particularly in $\varepsilon^{\prime}$. At this moisture content, the attenuation of the $8.9-\mathrm{cm}$ sample is $41.08 \mathrm{~dB}$, which is expected to be greater than the dynamic range of the experimental setup, and therefore the measurement error is expected to be large. In general, the $r^{2}$ value is lowest for the 2.5-cm samples solved using the approximate model (4).

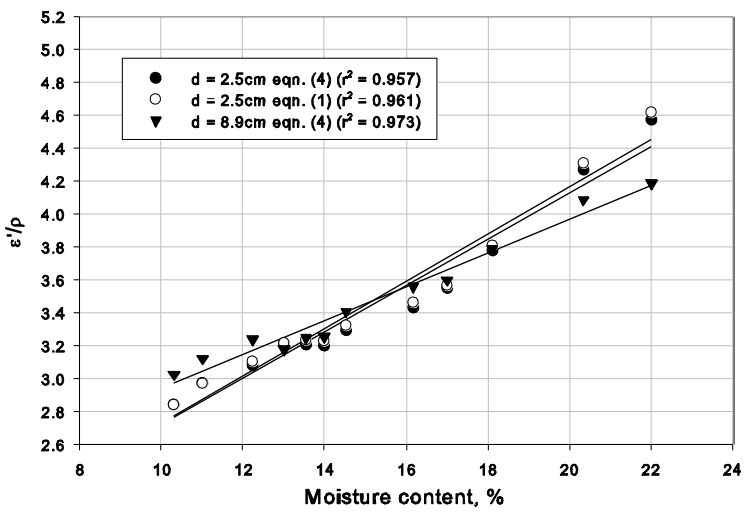

(a)

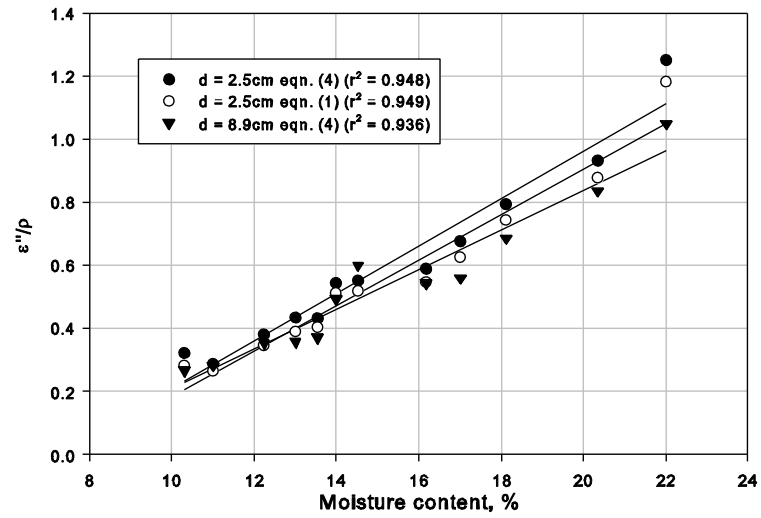

(b)

Figure 3: Variations of $\varepsilon^{\prime} / \rho$ (a) and $\varepsilon^{\prime \prime} / \rho$ (b) with moisture content for $2.5-\mathrm{cm}$ and $8.9-\mathrm{cm}$ grain samples at $10 \mathrm{GHz}$, determined with either (1) or (4).

\section{CONCLUSION}

A method has been presented for numerically solving a higher-order model for the free-space transmission equation used to determine the complex permittivity of thin grain samples. The ambiguity in the phase measurement has been accounted for by comparing several numerical solutions at two frequencies. Results for the permittivity of grain at five moisture contents for two sample thicknesses have been presented. It has been shown that the permittivity of relatively thin grain samples calculated by using the higher-order model is in good agreement with results calculated by using an approximate model, within the known ranges of validity of this approximate model. The advantage of using relatively thin grain samples is that a wider range of grain moistures can be reliably measured for a fixed sample thickness. 


\section{REFERENCES}

[1] E. Nyfors and P. Vainikainen, Industrial Microwave Sensors, Norwell, MA: Artech House, 1989.

[2] C. L. Pournaropoulos and D. K. Misra, "The co-axial aperture electromagnetic sensor and its application in material characterization," Meas. Sci. Technol.,vol. 8, pp. 1191-1202, 1997.

[3] S. Trabelsi, A. Kraszewski and S. O Nelson, "New Density-Independent Calibration Function for Microwave Sensing of Moisture Content in Particulate Materials," IEEE Trans. Instrum. Meas., vol. 47, no. 3, pp. 613-621, June 1998.

[4] J. Baker-Jarvis, E. J. Vanzura and W. A. Kissick, "Improved Technique for Determining Complex Permittivity with the Transmission/Reflection
Method," IEEE Trans. Microwave Theory Tech., vol. 38, 8, pp. 10961103, August 1990.

[5] R. Knöchel, W. Taute and C. Döscher, "Stray field ring resonators and a novel trough guide resonator for precise microwave moisture and density measurements," Meas. Sci. Techol., vol 18, pp. 1061-1068, 2007.

[6] S. Trabelsi, A. Kraszewski and S. O Nelson, "Phase-Shift Ambiguity in Microwave Dielectric Properties Measurements," IEEE Trans. Instrum. Meas., vol. 49, no. 1, pp. 56-60, February 2000.

[7] S. Trabelsi and S. O Nelson, "Free-space measurement of dielectric properties of cereal grain and oilseed at microwave frequencies," Meas. Sci. Tech. vol. 14, pp. 589-600, 2003. 